\title{
Compressif Giant Segmental Congenital Emphysema: Diagnosis and Traitment
}

\author{
Moussa Abdoulaye Ouattara ${ }^{1 *}$, Seydou Togo' ${ }^{1}$, Abdoul Aziz Diakité2, \\ Ibrahima Sankaré ${ }^{1}$, Bourama Kané2, Sekou Koumaré ${ }^{3}$, \\ Mody Abdoulaye Camara ${ }^{4}$, Zimogo Ziè Sanogo ${ }^{3}$, Sadio Yena ${ }^{1}$ \\ ${ }^{1}$ Department of Thoracic Surgery, Hospital of Mali, Bamako, Mali \\ ${ }^{2}$ Department of Pediatric, Gabriel Touré Teaching Hospital, Bamako, Mali \\ ${ }^{3}$ Department of Surgery, Point G Teaching Hospital, Bamako, Mali \\ ${ }^{4}$ Department of Imagery, Hospital of Mali, Bamako, Mali \\ Email: *zegouatt@yahoo.fr
}

Received 18 March 2016; accepted 15 April 2016; published 18 April 2016

Copyright (C) 2016 by authors and Scientific Research Publishing Inc.

This work is licensed under the Creative Commons Attribution International License (CC BY). http://creativecommons.org/licenses/by/4.0/

(c) (i) Open Access

\section{Abstract}

The giant congenital lobar emphysema is a rare malformation infant pathology. The authors report a similar case which is distinguished by its segmental location even rare with its compressive character in which segmentectomy was successful performed to lift emergency distress in a developing country.

\section{Keywords}

\section{Emphysema, Congenital, Segmentectomy}

\section{Summary}

Bronchopulmonary malformations (BPM) are resulting from development accidents of bronchopulmonary system. These are rare and polymorphic diseases including giant lobar emphysema congenital (GLE) are 3\% to $15 \%$ [1] [2]. Giant segmental congenital emphysema is even rare variant topography of the GLE, also progressing to respiratory distress. We report a case of delayed diagnosis of giant segmental congenital emphysema (GSE) to a compression stage with headquarters segmental which was intraoperative discovery. The treatment was performed in an emergency decompression by resection of the culmen in a developing country.

\footnotetext{
${ }^{*}$ Corresponding author.
}

How to cite this paper: Ouattara, M.A., Togo, S., Diakité, A.A., Sankaré, I., Kané, B., Koumaré, S., Camara, M.A., Sanogo, Z.Z. and Yena, S. (2016) Compressif Giant Segmental Congenital Emphysema: Diagnosis and Traitment. Surgical Science, 7, 195-198. http://dx.doi.org/10.4236/ss.2016.74027 


\section{Observation}

The family agreed the publication of this case report. This is a 4 month male, 3rd child of siblings, the result of a pregnancy during which ultrasounds performed did not find birth defects. It was addressed to the pediatric department tension pneumothorax. In its history there was a notion of dyspnea at birth without notion of fetal distress that evolved favorably by oxygen therapy. However small dyspnea crises associated with cough that occurred intermittent way from birth were treated medically. Moreover, the examination did not find any notion of malformation in the family. On admission the patient had respiratory distress with a respiratory rate of 28 cycles per minute, a room air oxygen saturation of $82 \%$, tachycardia at 130 beats per minute, a good general state with a $6 \mathrm{~kg}$ for size to $58 \mathrm{~cm}$. Physical examination revealed thoracic asymmetry, respiratory distress signs with chest indrawing, above and under sterna, a hyper-sounding on percussion and decreased vesicular murmurs in the ipsilateral auscultation. Chest radiography performed in front of the pediatric ward showed a homogeneous lucency with distension left hemithorax, decreased pulmonary vascular field and a shift of the mediastinum to the contralateral side (Figure 1). Chest computed tomography has clarified his left upper lobe topography (Figure 2). Bronchoscopy and lung scintigraphy were not performed. Left upper lobectomy was proposed and the preparation to the intervention was oxygen therapy, respiratory kinesitherapy with sessions of nebulized salbutamol and preoperative laboratory tests. The surgical procedure was performed under general anesthesia without selective intubation and the surgical approach was the left lateral thoracotomy. Intraoperatively we discovered a giant segmental emphysema depending on the culmen (Figure 3). The culminectomy was performed with periods of apnea to facilitate lung dissection (Figure 4). At the end of surgery, a good re-expansion of the lungula and lower lobe was obtained (Figure 5). Pathological examination macroscopically could not afford to reveal an obvious cause. However, microscopic examination has regained normal architecture of lung tissue with dilated alveoli. During his hospitalization, the patient presented a lung infection which has been treated medically with good evolution. He was discharged seven days in postoperative after the completion of a chest X-ray control (Figure 6). Currently to 6 months of the intervention it is an infant having good growth with satisfactory respiratory parameters.

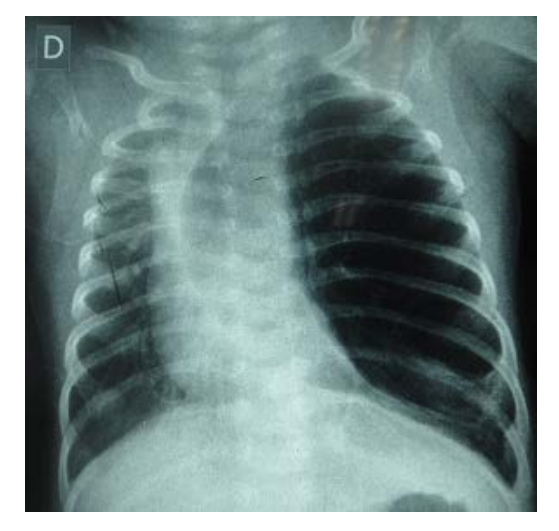

Figure1. Preoperative chest radiography.

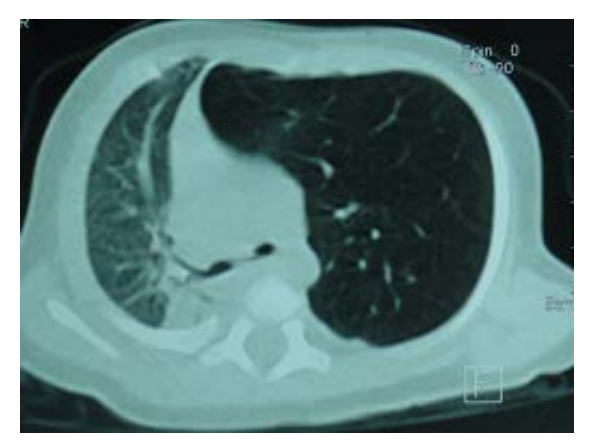

Figure 2. Preoperative chest computed tomography. 


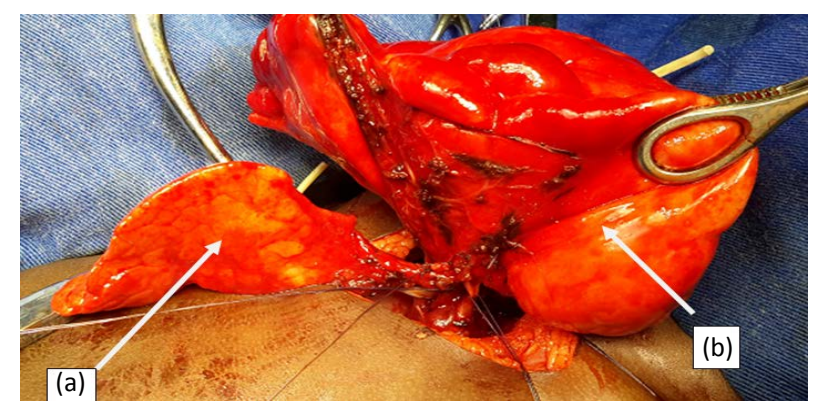

(a). Lingula

(b). Culmen

Figure 3. Intraoperative image.

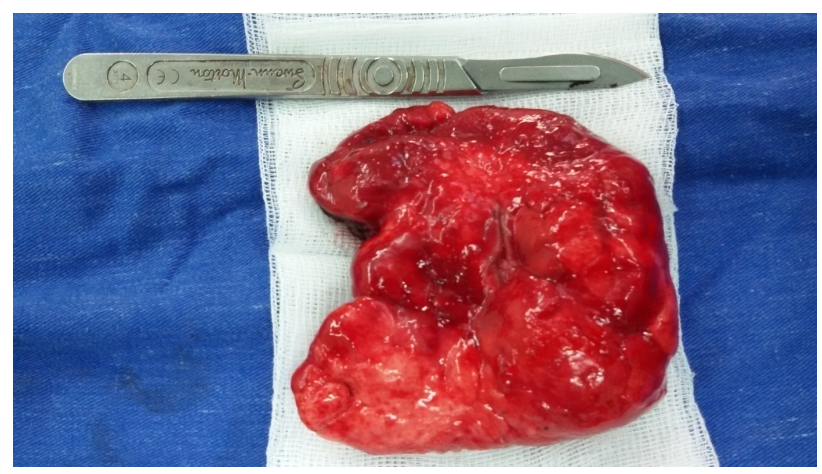

Figure 4. Surgical piece.

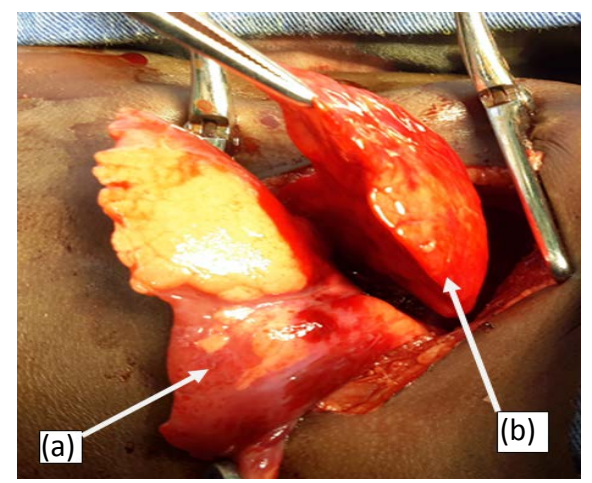

(a) Lower Lobe

(b) lingula

Figure 5. Lung reexpansion.

\section{Discussions}

This case is interesting to know because we did not find in the literature only one case reported, diagnosis is difficult preoperatively and it is a differential diagnosis with lobar emphysema giant t well documented.

The GSE is a rare topographic variation of the GLE. Prenatal diagnosis is rarely made ELG [1] [2]. This malformation is usually revealed in the first days or months of life [1]-[3]. Indeed, the demonstrations clinics appear at birth in $33 \%$ of cases and before the age of one month in $50 \%$ of cases [2] [3]. In our case, there was a misdiagnosis, the patient was treated for recurrent community-acquired pneumonia without radiological examination, hence the late discovery despite the early clinical signs. The late discovery is also related to the lack of specialized human resources in the country. Dyspnea is the most common clinical sign [3]. It is often gradual onset, evolving in a context of apyrexia, making evoke a malformation origin. In case of delayed diagnosis it can 


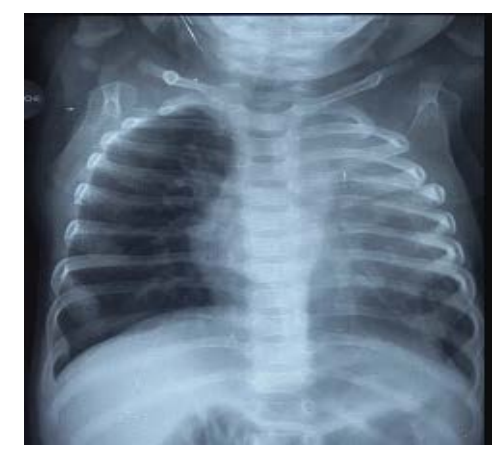

Figure 6. Postoperative chest radiography.

progress to respiratory distress by compression of adjacent structures, which can be life-threatening [3]. The diagnosis was suspected by chest radiography and confirmed by chest computed tomography. However the culminal seat was intraoperative diagnosis. This is explained by the importance of the distension that make difficult to identify the anatomical segmentation of the ipsilateral lung. Lung scintigraphy although is not available in our practice is an important consideration for viewing disorders of ventilation and perfusion at the lobe emphysema [2]. As bronchoscopy, it has an etiological and therapeutic interest but can be serious risk of increase hyperdistension. It eliminates the presence of an intra-bronchial foreign body, a mucus plug or look bronchial anomaly that may be responsible for emphysema. In this case respiratory distress has been a limit to its use. Our case is in $40 \%$ of cases where no etiology is found in the occurrence of over-distension [1] [3] [4]. A few patients of GLE have been described [1]-[3] but we found very little published in literature on this segmental topographic variation that has the same evolutionary risks. Because of the repercussions of the distended segment on mediastinum, ipsilateral and contralateral lung parenchyma, surgery appears to be the radical treatment because it helps to raise the compression [3] [5] [6]. It has the advantage of allowing anatomic resections therefore segmental parenchyma greater savings than the lobar form.

\section{Conclusion}

The segmental giant congenital emphysema is a topographic clinical form of the GLE with the same evolving risks and a similar support.

\section{Conflict of Interest}

None.

\section{References}

[1] Stocker, J.T. (1994) Congenital and Developmental Diseases. In: Dail, D.H. and Hammar, S.P., Eds., Pulmonary pathology, Springer-Verlag, Berlin, 155-181. http://dx.doi.org/10.1007/978-1-4757-3935-0_7

[2] Monin, P., Didier, F., Vert, P., et al. (1979) Giant Lobar Emphysema-Neonatal Diagnosis. Pediatric Radiology, 8, 259-260. http://dx.doi.org/10.1007/BF00974047

[3] Bouhaouala, M.H., Charfi, M.R., Tlili, K., et al. (1994) Giant Lobar Emphysema. The Cause of Compressive Hemithoracic Opacity in a Newborn Infant. Revue des Maladies Respiratoires, 11, 57-59.

[4] Raynaud, E.J., De Laguillaumie, B., Gaulme, J., et al. (1970) Anatomo-Clinical Study of a Case of Giant Lobar Emphysema in Newborn Infants. Journal Poumon et cœur, 26, 385-391.

[5] Desorgher, G., Bayart, M., Bayart, R., et al. (1970) Giant Lobar Emphysema in the Newborn. Left Superior Lobectomy. Journal des Sciences Médicales de Lille, 88, 39-45.

[6] Hall, N.J., Chiu, P.P. and Langer, J.C. (2015) Morbidity after Elective Resection of Prenatally Diagnosed Asymptomatic Congenital Pulmonary Airway Malformations. Pediatric Pulmonology, 2, 1-6. 